\title{
A methodology for LED placement in luminaires without lenses for optimal illumination of complex target areas
}

\begin{abstract}
Street lighting still faces some challenges, e.g., reducing power consumption, lowering light pollution, and making adaptive luminaires more effective among many other concerns. It is evident that the arrival of light emitting diodes (LEDs) in lighting systems has altered the state of the art and design strategies in lighting. In a traditional approach a single lamp, i.e., a single light point, is used. Then, by means of lenses, the specification of the radiation pattern is met. However, LED lamps allow for higher flexibility. It is possible to configure many small light sources, positioned and oriented at will. In this paper, a novel software tool able to provide the most suitable solution starting from a certain target lighting specification is proposed. This tool specifies the smallest number of LED required, their geometric orientation and power levels in order to comply with all the specifications. It is especially useful when trying to light up an irregular, twisted area, although this methodology can be adapted to any geometry. Furthermore, to validate the proposed methodology, two lamps were tested in the streets of Oviedo, Spain using a staggered arrangement.
\end{abstract}

Index Terms — LED lighting, Lighting Smart Grid, Smart Cities, Staggered arrangement, Street Lighting

\section{INTRODUCTION}

TREET lighting is experiencing a remarkable evolution due to the combination of LED lighting, renewable energy sources and Nenergy storage systems yielding a new concept called Lighting Smart Grids (LSG) [1], [2], [3]. They ought to be flexible, reliable and also efficient in order to accomplish the decisive requirement of energy efficiency [4]. The recent emergence of high-power LEDs brings new opportunities to modernize street lighting systems in our hometowns [5], [6]. LED luminaires are able to achieve high energy efficiency levels and lighting regulation capability [7], [8]. Besides, they have the potential to deliver precise beam patterns keeping a correct illuminance (E) and uniform light levels, minimizing light pollution and improving glare reduction [9], [10]. LEDs have deeply changed in recent years and therefore, so have lamps. These new LEDs endow lamps with the possibility of guiding the light beam where needed, without lenses and matching all the specifications. In addition, each lighting point can be easily regulated with the help of today's silicon devices.

There are diverse software tools (e.g. Calculux, Relux or DIALux [11]) that provide solutions to designers based on the indoor/outdoor target region, current available lamps in the market, and illumination requirements. The common solution for street lighting is the one depicted in Fig. 1a: a lamppost with the LEDs placed horizontally facing the ground, covered by a lens used to distribute the light beams in the required directions [12], [13]. To offer an alternative solution to this problem, this paper proposes a parametric software tool which helps to decide the correct geometric distribution and orientation of each group of LEDs and their required power. It provides a solution that forgoes any kind of lens, although it could be placed to enhance the behavior of the luminaire. This tool complies with all illuminance specifications and it is especially focused on street lighting with curved and twisted shapes. In addition, it allows customizing different light pattern diagrams for various street scenarios, e.g., a corner, a roundabout, an intersection and many more. Fig. 1b shows a possible solution given by this tool: LEDs are placed with a certain orientation in order to properly light the target area since there is no lens. However, as depicted, an outer shell protects electronics and sensitive devices from weather conditions, as in common street luminaires.

Moreover, this methodology is especially useful to take advantage of smart lighting systems. For example, the user can easily change the radiation pattern of the luminaire, dim the LEDs, or turn some groups of LED off. A prior version of this work has been presented in [14]. That work addressed the design methodology from a different point of view, being more difficult to understand.

In the following sections, the proposed methodology is going to be explained and studied in detail: in Section II, the mathematical background of the led positioning tool is shown. Then, a practical example based on the street lamps installed in Oviedo, Spain, is detailed in Section III. Section IV deals with experimental results that validate the proposed methodology. Finally, in Section V the conclusions of this work and some future developments are exposed. 

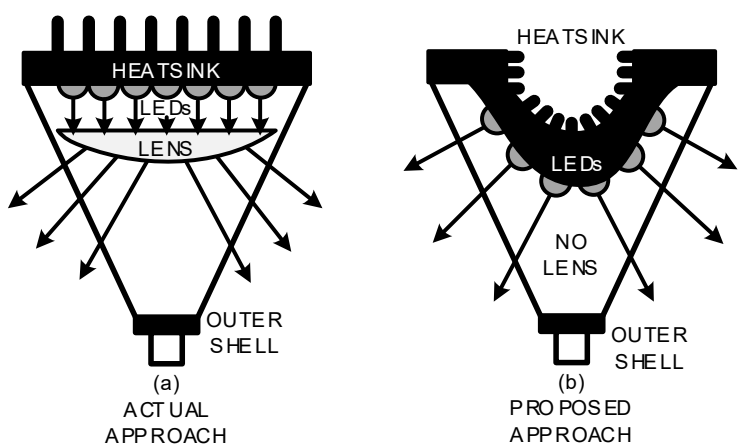

Fig. 1. Comparison among street lampposts: (a) Today's usual approach with power LEDs and lenses vs. (b) a proposed approach without lens.

\section{DESCRIPTION OF THE PROPOSED METHODOLOGY}

The developed software tool for the geometric LED positioning (GLP tool) carries out three steps: 1) Define the target region. 2) Divide the target region into smaller subregions to obtain the proper position and direction of the LED arrays (as depicted in Fig. 1b). 3) Calculate the power required to light up each subregion considering a target luminous flux. These steps are described in the following subsections:

\section{A. Step 1: define the target region}

The target region that has to be illuminated can be symmetric or not. The GLP tool is equally effective regardless the shape of the target area.

Fig. 2 illustrates a strange but plausible target region which shall serve to explain how the proposed methodology works. As depicted, $L P$ is the height of the lamppost and $D$ is the ground distance from the base of the street lamp to any point ( $D_{M A X}$ in the case of the boundary). Several parameters have to be previously defined in order to operate in polar coordinates: first, the angle $\Omega$ which goes all the way round the perimeter of the target region. This angle is defined counterclockwise. Second, the light angle $(\beta)$, is the angle between any point of the target area and the luminaire upon the $z$ axis. This angle is a function of $\Omega, D$, and $L P$. The maximum value of the light angle $\left(\beta_{M A X}\right)$ corresponds to the one that points to the boundary of the target region.

\section{B. Step 2: divide the target area into smaller subregions}

Herein, the target region is divided into a finite number of subregions. Each subregion can be defined by two angles: $\Omega_{\mathrm{S}}$ and $\beta_{\mathrm{sf} .}$ These angles aim at the center of the selected area, as depicted in Fig. 3.

However, the target region should be split in a more suitable way, as shown in Fig. 4a. In this case, each subregion belongs to a single strip/sector.

As a first approach, the target area could be divided into three strips or rings $(n f=3)$ and ten sectors $(n s=10)$, i.e., 30 subregions. In the same way, three boundaries appear based on the light angle $\beta$. Once the maximum value of this angle is calculated, $\beta_{\max }$, those three fringes can be assumed as: $\beta_{\max }, \frac{2 \cdot \beta_{\max }}{3}, \frac{\beta_{\max }}{3}$ although this is not a mandatory division.

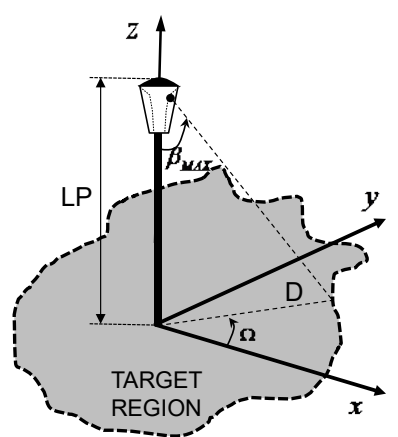

Fig. 2. Target region over $x y$ coordinates showing the parametric angles $\Omega$

$$
\text { and } \beta_{M A X} \text {. }
$$




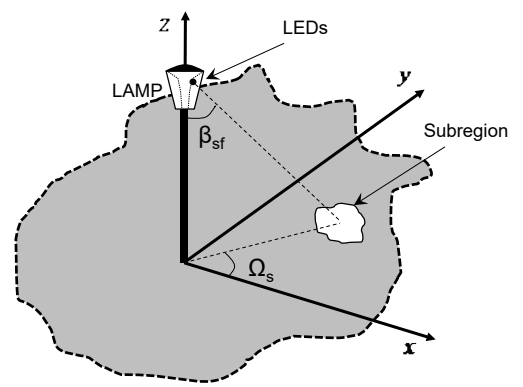

Fig. 3. Example of a small subregion within the target area. The sum of all the subregions would give the whole target area.

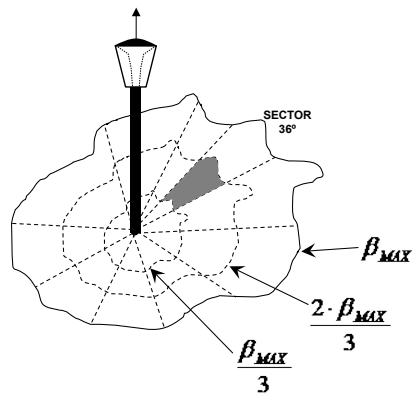

(a)

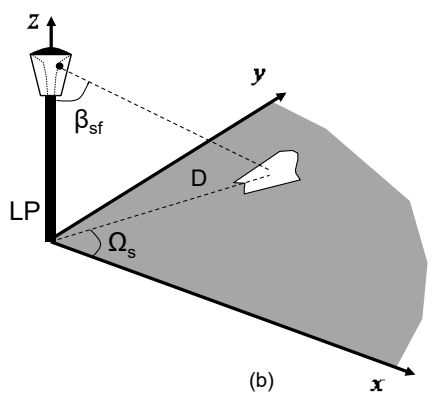

Fig. 4. (a) Division of the target region in 30 divisions. (b) $\Omega_{\mathrm{s}}$ and $\beta_{\mathrm{sf}}$ angles for a particular subregion.

The value of the angles $\Omega_{\mathrm{s}}$ and $\beta_{s f}$ for each subregion can be obtained from (1) and (2):

$$
\begin{gathered}
\Omega s(s)=\frac{360}{n s} \cdot\left(s-\frac{1}{2}\right) \\
\beta_{s f}(s, f)=\frac{\beta_{\max }(\Omega s(s))}{n f} \cdot\left(f-\frac{1}{2}\right)
\end{gathered}
$$

where $s \in[1, n s]$ and $f \in[1, n f] . \Omega_{\mathrm{S}}$ depends on the sector whereas $\beta_{s f}$ depends both on the sector and the strip.

As mentioned before, both angles $\Omega_{\mathrm{s}}$ and $\beta_{s f}$ aim at the center of the subregion and thus they are key parameters for the whole design. They provide the most suitable position and tilt of the LEDs in order to light each subregion. However, a third step is required to define how much electrical power is required to illuminate every subregion according to the specifications.

\section{Step 3: calculation of the power required to illuminate each subregion}

Provided that the position and tilt of the LEDs are known, the methodology has to face the calculation of the electrical power and therefore the luminous flux to properly light up the target area. In order to obtain the total luminous flux $(\Phi)$ or the total power $(P)$ required for the application to cover the whole target area, two key variables need to be introduced: the Angular Flux Density $\left(\Phi_{\beta}\right)$ and the Angular Power Density $\left(P_{\beta}\right)$. These variables refer to the luminous flux and the power that are pointed to a certain subregion in the target area. However, to obtain these variables, a previous mathematical development has to be presented.

Let us consider a differential of area inside one of the target subregions $\left(d A_{s f}\right)$ presented in II.B. This $d A_{s f}$ is shown in Fig. 5 and it is defined by both a differential of $\beta_{s f}$ and $\Omega_{\mathrm{s}}$. The relationship between $d A_{s f}$ and these design parameters is:

$$
d A_{s f}=\left(\frac{\pi}{180}\right)^{2} \cdot L P^{2} \cdot \tan \left(\frac{\pi}{180} \cdot \beta_{s f}\right) \cdot\left[1+\left(\tan \left(\frac{\pi}{180} \cdot \beta_{s f}\right)\right)^{2}\right] \cdot d \beta \cdot d \Omega
$$

Once $d A_{s f}$ is known, the differential of solid angle $\left(d S A_{s f}\right)$ from the lamp point of view can be defined. Its expression also depends on the distance from the lamp to the differential of area: 


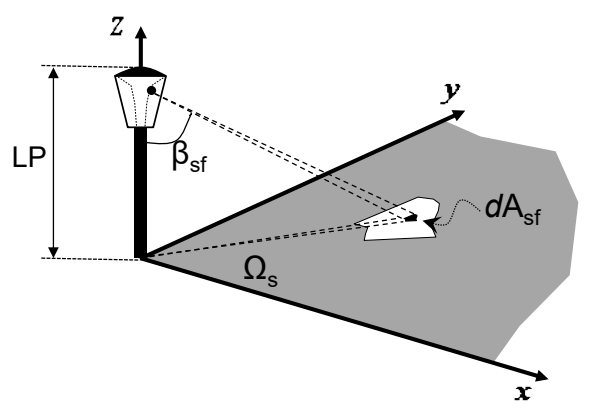

Fig. 5. Differential of area $\left(d A_{s f}\right)$ over a differential of light angle $\left(d \beta_{s f}\right)$ and differential of $\Omega_{\mathrm{s}}\left(d \Omega_{\mathrm{s}}\right)$.

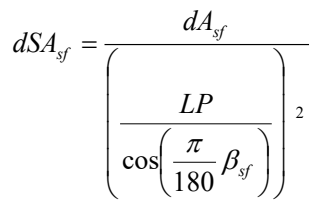

Thus, combining the target illuminance requirement (E, in lx) with (3) and (4), the expression of the differential of angular flux density upon the center of a generic subregion can be obtained:

$$
d \Phi(\beta)=E \cdot \frac{\left(\frac{\pi}{180}\right)^{2} \cdot L P^{2} \cdot \tan \left(\frac{\pi}{180} \cdot \beta_{s f}\right) \cdot\left[1+\left(\tan \left(\frac{\pi}{180} \cdot \beta_{s f}\right)\right)^{2}\right]}{\cos \left(\frac{\pi}{180} \cdot \beta_{s f}\right)} \cdot d \beta \cdot d \Omega
$$

The expression of the differential of angular power density $\left(d P_{\beta}\right)$ is derived from (5) by taking into account three kinds of efficiencies: LEDs luminous efficiency $\left(\eta_{\mathrm{L}}\right.$ in $\left.1 \mathrm{~m} / \mathrm{W}\right)$, the electronic driver efficiency ( $\eta_{\mathrm{E}}$ in p.u.) and the optical one of the outer shell ( $\eta_{\mathrm{O}}$ in p.u.). Hence, $d P_{\beta}$ can be written as follows:

$$
d P_{\beta}(\beta)=E \cdot \frac{\left(\frac{\pi}{180}\right)^{2} \cdot L P^{2} \cdot \tan \left(\frac{\pi}{180} \cdot \beta_{s f}\right) \cdot\left[1+\left(\tan \left(\frac{\pi}{180} \cdot \beta_{s f}\right)\right)^{2}\right]}{\cos \left(\frac{\pi}{180} \cdot \beta_{s f}\right) \cdot \eta_{L} \cdot \eta_{E} \cdot \eta_{O}} \cdot d \beta \cdot d \Omega
$$

The total power required to illuminate a whole subregion comes from integrating (6) along the length of the strip as well as the angles that delimitate its sector. Hence, the general expression of the electrical power required for a group of LEDs to light up a certain subregion is noted in (7). In addition, (7) can be graphically described as in Fig. 6.

$$
\begin{aligned}
d P_{s f}(s, f)=d P_{\beta}(\beta) \cdot d \beta \cdot d \Omega \rightarrow & \\
P_{s f}(s, f) & =\int_{\frac{360 \cdot(s-1)}{n s}}^{\frac{360 \cdot s}{n s}} \frac{\beta_{\max }\left(\frac{\beta_{\max }(\Omega) \cdot(f-1)}{n f}\right.}{n f} P_{\beta}(\beta) \cdot d \beta \cdot d \Omega
\end{aligned}
$$

\section{Lamp's total power}

While the sum of each $P_{s f}$, i.e., the sum of the power of every group of LEDs, gives the total power required to illuminate the target area under study, it can also be calculated by (8). This expression of the total power is the double integral of the angular power density between the surface defined by $\beta_{\max }$ and $\Omega$ :

$$
P_{L A M P}=\int_{0}^{360} \int_{0}^{\beta \max (\Omega)} P_{\beta}(\beta) \cdot d \beta \cdot d \Omega
$$

In summary, the inputs of the proposed methodology are itemized in Fig. 7. The user has to define the perimeter of the target 
region, the height of the lamppost, the target illuminance and finally, how the target region is going to be split (number of sectors and strips). The outputs are the position and tilt of the LEDs, the power required in each subregion and therefore, the minimum number of LEDs needed to illuminate it, and finally, the lamp's total power.

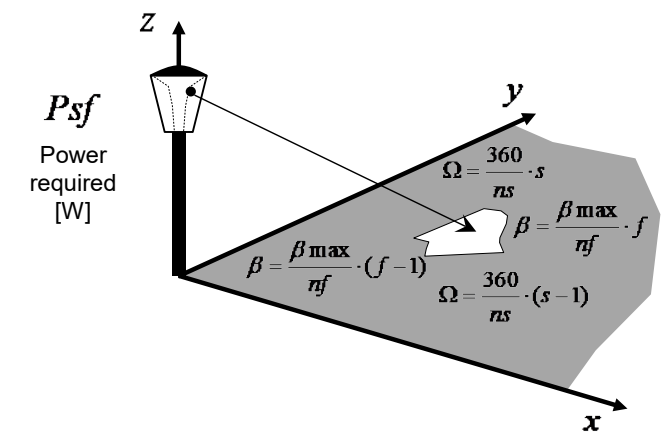

Fig. 6. Calculation of the power required to illuminate one subsector of the target region in compliance with a certain illuminance requirement.

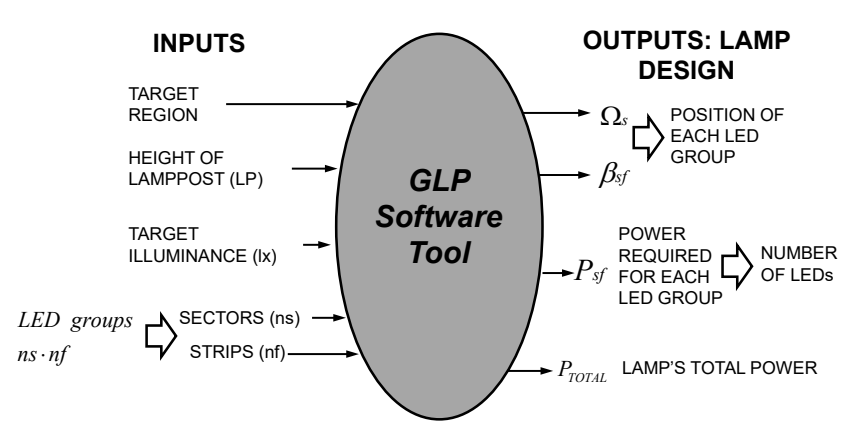

Fig. 7. Inputs and outputs of the GLP software tool.

\section{PRACTICAL IMPLEMENTATION OF THE METHODOLOGY ON A REAL TARGET REGION}

Fig. 8 shows the typical structure of a bilateral staggered lighting system. The lampposts are evenly spaced and they light a particular area over both the road and the sidewalk.

By courtesy of the city council of Oviedo, the authors have studied and proposed a solution to a real case, as depicted in Fig. 9. In order to ease the representation of the target area, an $x y z$ coordinates system has been also included. Herein, the width of the road is denoted as $\mathrm{R}$ whereas the sidewalk is represented by $\mathrm{S}$. Both the road and the sidewalk have been divided into two symmetric regions around the $x$-axis: Road Left (RL), Road Right (RR), Sidewalk Left (SL), and Sidewalk Right (SR). The height of the streetlamp is LP. On the other hand, H can be between one and thrice LP in staggered designs.

As it may be seen, this is a complex target lighting region. The proposed methodology must provide an accurate LEDs distribution in each lamppost in order to light the required area. There must be a flawless matching among the staggered arrangement and an independent control of the illumination levels in the target zones. Moreover, typical street lighting specifications, besides appearance, are: reduced glare, improved eye comfort, homogeneity and last but not least, a proper illuminance level (E). In this particular work, the authors have followed city council directives. Therefore, E is about 25 lx and should be provided with the lowest possible power levels.

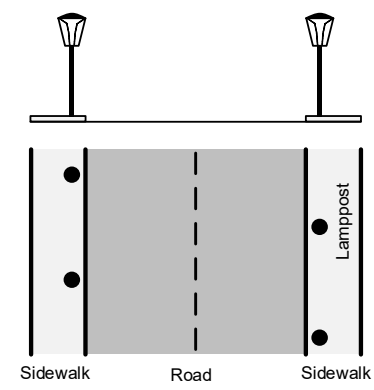

Fig. 8. Classic bilateral staggered configuration. 


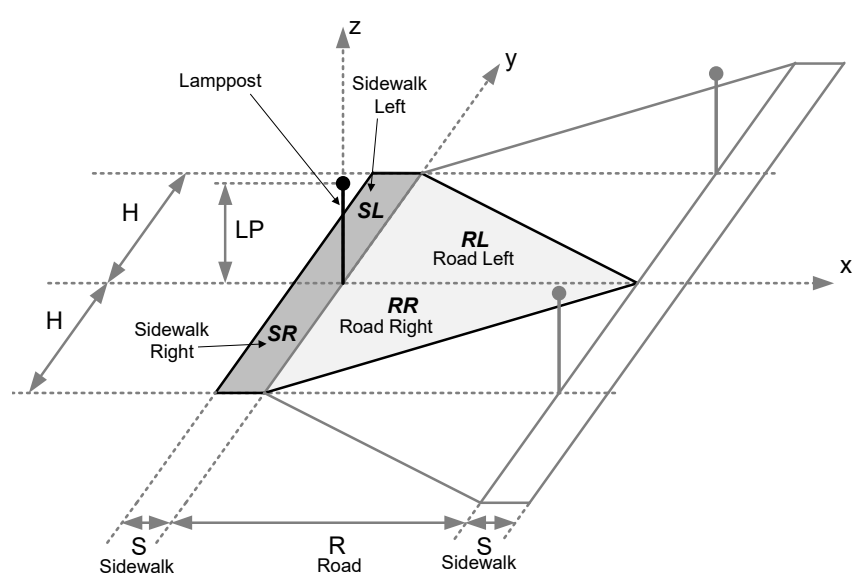

Fig. 9. Street lighting in staggered configuration. A real case in Oviedo, Spain.

The aforementioned steps of Section II were followed for this particular case. First of all, the target region boundary is detailed based on the following dimensions: $H=10 \mathrm{~m}, R=9 \mathrm{~m}, S=2 \mathrm{~m}$, and $L P=5 \mathrm{~m}$.

The target region in geometrical dimensions depends only on $\Omega$. Each point of the boundary $\left(D_{\max }\right)$ is represented over a polar diagram in Fig. 10a. On the other hand, the target region can be also depicted in angular dimensions $(\Omega, \beta)$. This graphical representation can be found in Fig. 10b based upon (9):

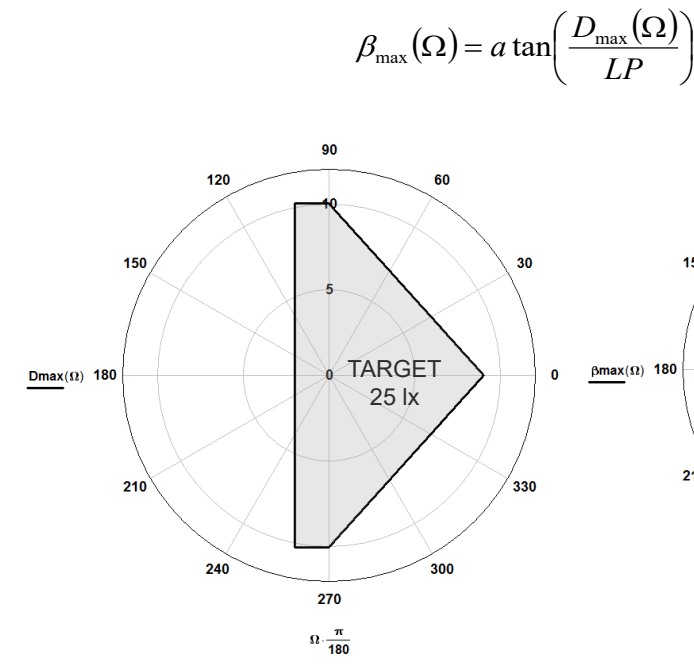

(a)

Fig. 10. (a) Target region in geometrical dimensions. (b) Target region in angular dimensions.

As explained in Subsection II.B, the following step is dividing the target region into smaller subregions. In this case, we have decided to split the area into 12 sectors and 3 strips. The division of the target region in angular dimensions can be found in Fig. 11 along with the nomenclature.

Once all the required inputs are known, the first output can be obtained i.e., the tilt of the LEDs. From (2) and taking into account that each $\beta_{s f}$ points to the center of the correspondent subregion, Table I can be filled out.

In addition, (7) yields Table II, which gathers the power required to illuminate each subregion. The total power of the lamp, $56.9 \mathrm{~W}$, is also shown. 


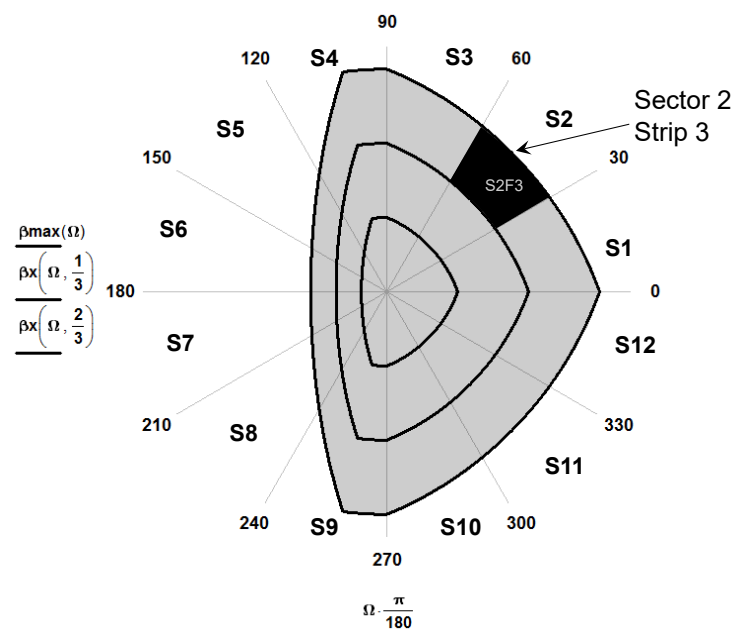

Fig. 11. Division of the target area in 36 subregions in angular dimensions $(\Omega, \beta)$.

TABLE I

ADEQUATE POSITION OF THE LEDS BY SECTOR AND STRIPE

\begin{tabular}{|c|c|c|c|c|c|c|c|c|c|c|c|c|c|c|}
\hline \multirow{2}{*}{\multicolumn{3}{|c|}{$\begin{array}{l}\text { ANGULAR POSITION OF } \\
\text { LEDs ARRANGEMENTS }\end{array}$}} & \multicolumn{12}{|c|}{ SECTOR(ns) } \\
\hline & & & 1 & 2 & 3 & 4 & 5 & 6 & 7 & 8 & 9 & 10 & 11 & 12 \\
\hline \multicolumn{3}{|c|}{$\Omega$ s [degrees] } & 15 & 45 & 75 & 105 & 135 & 165 & 195 & 225 & 255 & 285 & 315 & 345 \\
\hline & & & & & & & & & & & & & & \\
\hline \multirow{3}{*}{$\beta$ sf [degrees] } & \multirow{3}{*}{$\operatorname{STRIP}(\mathrm{nf})$} & 1 & 9.4 & 8.9 & 9.7 & 9.5 & 4.9 & \begin{tabular}{|l|}
3.7 \\
\end{tabular} & 3.7 & 4.9 & 9.5 & \begin{tabular}{|l|}
9.7 \\
\end{tabular} & 8.9 & 9.4 \\
\hline & & 2 & 28.2 & 26.6 & 29.0 & 28.5 & 14.7 & 11.2 & 11.2 & 14.7 & 28.5 & 29.0 & 26.6 & 28.2 \\
\hline & & 3 & 46.9 & 44.4 & 48.3 & 47.6 & 24.6 & \begin{tabular}{|l|}
18.7 \\
\end{tabular} & 18.7 & 24.6 & 47.6 & 48.3 & 44.4 & 46.9 \\
\hline \multicolumn{3}{|c|}{ [degrees] } & 56.3 & 53.3 & 57.9 & 57.1 & 29.5 & 22.5 & 22.5 & 29.5 & 57.1 & 57.9 & 53.3 & 56.3 \\
\hline
\end{tabular}

TABLE II

POWER REQUIRED BY EACH LED ARRANGEMENT

\begin{tabular}{|c|c|c|c|c|c|c|c|c|c|c|c|c|c|c|}
\hline \multirow{2}{*}{\multicolumn{3}{|c|}{$\begin{array}{l}\text { POWER REQUIRED BY LED } \\
\text { ARRANGEMENT }\end{array}$}} & \multicolumn{12}{|c|}{ SECTOR (ns) } \\
\hline & & & 1 & 2 & 3 & 4 & 5 & 6 & 7 & 8 & 9 & 10 & 11 & 12 \\
\hline \multirow{3}{*}{ Psf [W] } & \multirow{3}{*}{ STRIP (nf) } & 1 & 0.2 & 0.2 & 0.3 & 0.2 & 0.1 & 0.0 & 0.0 & 0.1 & 0.2 & 0.3 & 0.2 & 0.2 \\
\hline & & 2 & 1.1 & 0.9 & 1.2 & 1.1 & 0.2 & 0.1 & 0.1 & 0.2 & 1.1 & 1.2 & 0.9 & 1.1 \\
\hline & & 3 & 5.4 & 3.8 & 6.5 & 6.5 & 0.4 & 0.2 & 0.2 & 0.4 & 6.5 & 6.5 & 3.8 & 5.4 \\
\hline
\end{tabular}

\begin{tabular}{|c|c|c|c|c|c|c|c|c|c|c|c|c|}
\hline Psector [W] & 6.7 & 4.9 & 8.0 & 7.8 & 0.7 & 0.4 & 0.4 & 0.7 & 7.8 & 8.0 & 4.9 & 6.7 \\
\hline Ptotal [W] & \multicolumn{10}{|c|}{56.9} \\
\hline
\end{tabular}

From Tables I and II, some conclusions about the design procedure can be obtained:

1. The power required to illuminate the closest subregions to the lamppost is rather low. Those subregions that belong to strip 1 could be bigger in order to handle more power.

2. The third strip i.e., the furthest one from the street lamp needs higher amounts of power which in the end means more LEDs. It would be a good idea to split these subregions into smaller areas and increase the number of strips in order to not concentrate so much heat in that part of the lamp/heat sink.

\section{LABORATORY PROTOTYPE}

In order to validate the methodology, a laboratory prototype has been built. The main goal of the prototype is to prove it is possible to achieve lux uniformity in a certain target area without using any lens through the correct LED positioning. Also, the independent power control capability of each LED arrangement is going to be validated in order to improve this illuminance homogeneity.

In Fig. 12, an example of one branch of the lamp prototype that lights all the strips within a single sector is shown. The branch is bent at different angles according to the required geometrical orientation given by the GLP tool. An aluminum structure serves as heat sink as well as the base to fix the LEDs to. The laboratory lamp used to carry out all the experiments is shown in Fig. 13. 


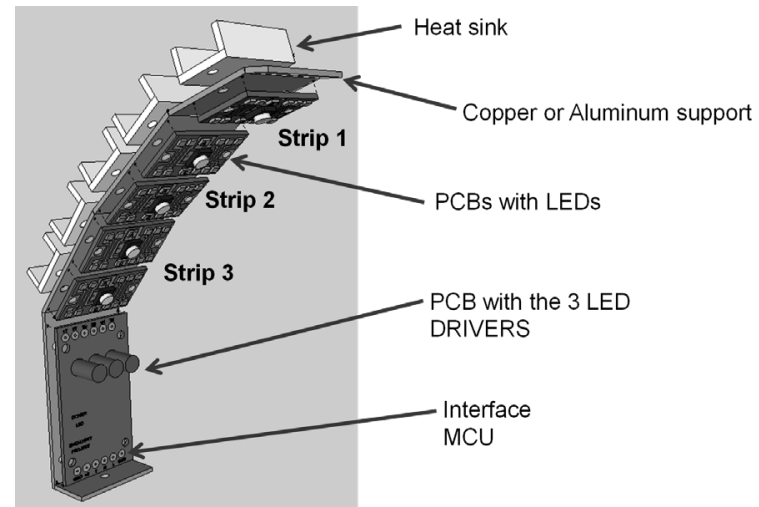

Fig. 12. Prototype of one branch of the street lamp.
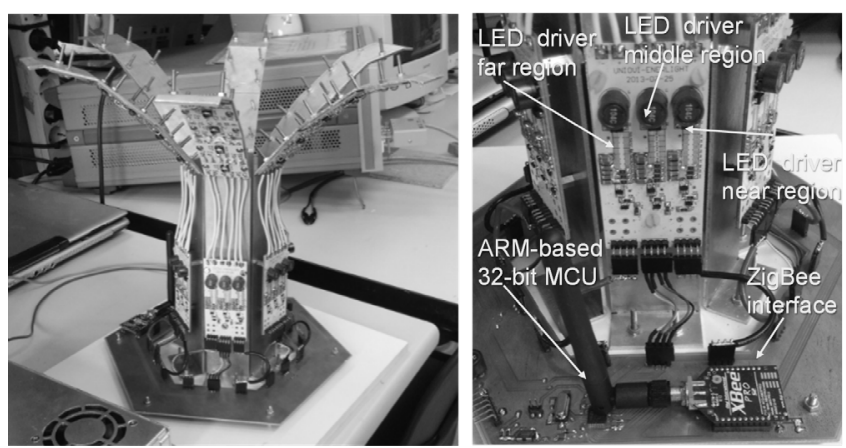

Fig. 13. Lamp prototype under study.

Each branch is meant to uniformly light up a sector by means of three different light angles. It consists of five LEDs with their respective drivers and the heat sink. The whole lamp, i.e., all the lamp branches, are controlled by a single 32-bit ARM-based microcontroller unit (MCU) with ZigBee connectivity.

The following is a more detailed explanation of the elements that form the lamp.

\section{A. LEDs}

This laboratory prototype uses Cree Xlamp MC-E LEDs. Each chip has four individual LED inside that can be connected at will owing to its modular structure. This model is rather old and thus its efficiency is not as high as today's LEDs. Nowadays manufacturers are able to supply power LEDs with efficiencies close to $200 \mathrm{~lm} / \mathrm{W}$ [15], [16].

\section{B. LED driver}

The power LED modules are electrically supplied by low-cost drivers: ZXLD1362 from ZETEX®. This driver allows a wide input voltage range providing a stable output current that depends upon a shunt resistor. The current delivered to the LEDs can be modified by a PWM signal that would come from the MCU to the ZETEX IC.

\section{Heat sink}

Fig. 12 shows details of laboratory assembly using a custom made double side-PCB for each LED module to ease the attachment over an aluminum structure. It allows heat dissipation as well as setting the angular light angle $(\beta)$ required to light up the target area.

\section{D. $M C U$}

A low-cost ARM-based 32-bit MCU from ST (STM32F051R8) has been used in order to implement the PWM control in all drivers. The MCU incorporates ZigBee communications capability and full customization of the illumination level required in each point of the target area.

The lamp prototype has been tested in the laboratory to analyze the homogeneity of the light over the target region. This prototype is shown in Fig. 13. The lamp under test has been placed $5 \mathrm{~m}$ height whereas the target region has been split into two $10^{\circ}$ sectors and six strips separated $1 \mathrm{~m}$ as depicted in Fig. 14.

Two different tests have been carried out to obtain a homogeneous illuminance of $25 \mathrm{~lx}$, as gathered in Table III. In the first test all the LEDs were at maximum power, i.e., there was no external regulation from the MCU. As it can be seen, the illuminance levels are quite different. However, in the second test and according to the outputs given by the methodology, an 
external regulation with the MCU has been included and a more uniform illuminance was obtained. Strips 1 and 2 required a $45 \%$ illuminance reduction whereas strips 3 and 4 a 40\%. Owing to the distance from the lamppost, LEDs from strips 5 and 6 have not needed any power regulation.

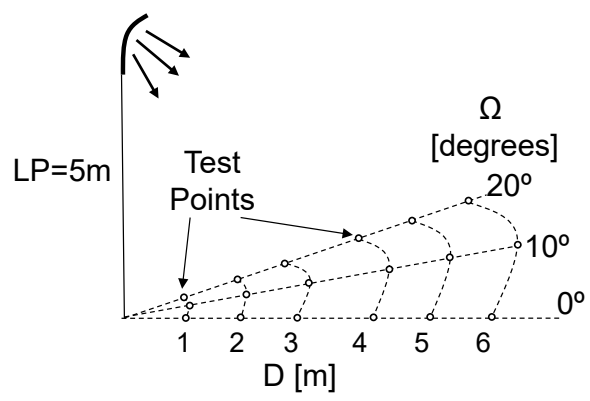

Fig. 14. Light uniformity test of the lamp prototype.

Although this homogeneity could be improved with the help of lenses, Table III proves that the methodology is valid. It is possible to illuminate a complex target region with a proper positioning of the LEDs and by controlling their electrical power without the use of external lenses.

\section{CONCLUSIONS}

In this paper, a methodology that decides how to properly distribute LED arrangements in the lamp in terms of position, tilt, and electrical power was proposed. This methodology was implemented as a software parametric tool that provides a solution based upon the target area the user wants to illuminate. This solution does not require the use of lenses to uniformly distribute the light although they could be added to the luminaire in order to enhance its behavior. The proposed methodology uses the concept of sending the light beams in the correct direction with specific angle and intensity.

This methodology was applied to a real complex target region and a prototype lamp was built in the laboratory to evaluate the proposed concepts. This prototype provides light regulation from 0 to $100 \%$ for adjusting the light level requirement with an MCU. It also provides dimming capability according to external environmental conditions.

Several experimental design procedures have been proposed according to different lighting strategies and real target regions (e.g., Oviedo, Spain).

As future developments, a new prototype with the current LEDs in the market could be built. This new lamp would have more LED arrangements allowing a fine-grained control. In addition, the structure can be redesigned in order to facilitate its construction.

TABLE III

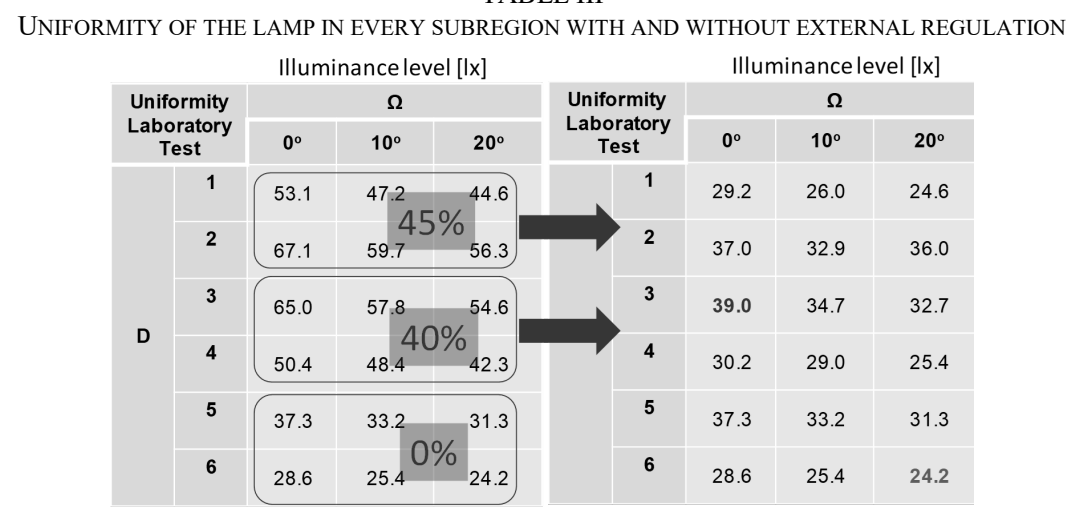

Further, we are currently working on customizable lighting configurations. This feature is a difficult task to accomplish with conventional designs due to the fact that more than one radiation pattern is not achievable by means of the same lens. However, with the proposed methodology and a hierarchical control, different light patterns can be generated. Some of these lighting configurations can be checked on Fig. 15.

\section{ACKNOWLEDGMENT}

This work has been supported by the Ministry of Economy and Competitiveness of the Government of Spain (MINECO), the Government of the Principado de Asturias and the European Union trough the European Regional Development Fund (ERFD), 
under Research Grants ENE2013-41491-R (LITCITY Project) and GRUPIN14-076.

The authors would also like to thank the Workroom on Renewable Energy (WRE) magazine staff their altruistic help during the brainstorming and building all the prototypes.

And last but not least, thanks to MEng. Carlos Garcia Gonzalez, Head of the Lighting Section and Electric Installations in the Oviedo's city council for their valuable recommendations and advice.

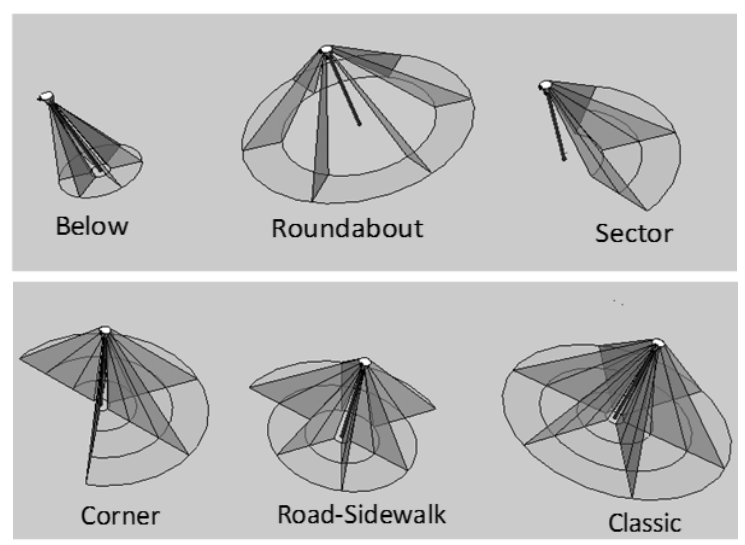

Fig. 15. Customizable lighting configurations.

\section{REFERENCES}

[1] M. Jaureguizar, M. Rico-Secades, E. L. Corominas, D. Garcia-Llera and A. J. Calleja, "Enerlight project: Walking from electronic lighting systems to Lighting Smart Grid," 2013 IEEE International Conference SmartMILE, Gijon, 2013, pp. 1-6.

[2] J. Cardesín, D. García-Llera, E. López-Corominas, A. J. Calleja, J. Ribas and D. Gacio, "Low cost intelligent LED driver for public Lighting Smart Grids," 2013 IEEE International Conference SmartMILE, Gijon, 2013, pp. 1-6.

[3] Tursunov, M. N.; Mirzabaev, A. M.; Dadamukhamedov, S.; Kanonerov, V. P.; Abdullaev, E. \& Tukfatullin, O. F. Photoelectric device for street lighting based on silicon solar cells Applied Solar Energy, 2009, 45, 17-20

[4] M. F. de Melo, W. D. Vizzotto, P. J. Quintana, A. L. Kirsten, M. A. Dalla Costa and J. Garcia, "Bidirectional Grid-Tie Flyback Converter Applied to Distributed Power Generation and Street Lighting Integrated System," in IEEE Transactions on Industry Applications, vol. 51, no. 6, pp. 4709-4717, Nov.-Dec. 2015.

[5] K. J. Gaston, "Sustainability: A green light for efficiency," Nature, vol. 497, pp. 560-561, 2013.

[6] X. Long, R. Liao and J. Zhou, "Development of street lighting system-based novel high-brightness LED modules," in IET Optoelectronics, vol. 3, no. 1, pp. 40-46, February 2009.

[7] J. M. Alonso, J. Vina, D. G. Vaquero, G. Martinez and R. Osorio, "Analysis and Design of the Integrated Double Buck-Boost Converter as a HighPower-Factor Driver for Power-LED Lamps," in IEEE Transactions on Industrial Electronics, vol. 59, no. 4, pp. 1689-1697, April 2012.

[8] D. Gacio, J. M. Alonso, A. J. Calleja, J. Garcia and M. Rico-Secades, "A Universal-Input Single-Stage High-Power-Factor Power Supply for HB-LEDs Based on Integrated Buck-Flyback Converter," in IEEE Transactions on Industrial Electronics, vol. 58, no. 2, pp. 589-599, Feb. 2011.

[9] C. C. Sun et al., "Design of LED Street Lighting Adapted for Free-Form Roads," in IEEE Photonics Journal, vol. 9, no. 1, pp. 1-13, Feb. 2017.

[10] Keitz H.A.E. "Light Calculations and Measurements". N.V. Philips. 1974. ISBN: 84-283-0576-5

[11] Philips lighting simulation software: http://www.lighting.philips.com/main/support/support/dialux-and-other-downloads

[12] Pizzuti, S.; Annunziato, M. \& Moretti, F. Smart street lighting management Energy Efficiency, 2013, 6, 607-616

[13] Lévai, B. L. \& Bánhelyi, B. Fasano, G. \& Pintér, Já. D. (Eds.) Automatic Design of Optimal LED Street Lights. Optimized Packings with Applications, Springer International Publishing, 2015, 175-185

[14] D. Garcia-Llera et al., "Optimizing LED lamps design for street lighting with staggered arrangement allowing energy saving strategies in a Lighting Smart Grid context," in IEEE Industry Application Society - 51st Annual Meeting, IAS 2015, Conference Record, 2015.

[15] Cree ${ }^{\circledR}$ XLamp® XP-G3 LED: http://www.cree.com/led-components/media/documents/dsXPG3.pdf

[16] Cree ${ }^{\circledR}$ XLamp® XHP70.2 LEDs: http://www.cree.com/led-components/media/documents/ds-XHP702_1.pdf 